\title{
Successful wire-guided fiberoptic nasotracheal intubation in a patient with hemifacial microsomia - A case report -
}

Received May 16, 2019

Revised 1st, July 22, 2019

2nd, August 23, 2019

Accepted September 3, 2019

\section{Corresponding author}

Bon-Nyeo Koo, M.D., Ph.D.

Department of Anesthesiology and

Pain Medicine, Anesthesia and

Pain Research Institute, Severance

Hospital, Yonsei University College

of Medicine, 50-1 Yonsei-ro,

Seodaemun-gu, Seoul 03722, Korea

Tel: 82-2-2228-2420

Fax: 82-2-312-7185

E-mail: koobn@yuhs.ac

\section{ORCID}

https://orcid.org/0000-0002-3189-1673

\section{Seon Ju Kim ${ }^{1}$, Haeyeon Lee ${ }^{1,2}$, Seung Yeon Choi ${ }^{2}$, and Bon-Nyeo Koo ${ }^{2}$}

Department of Anesthesiology and Pain Medicine, ${ }^{1}$ National Health Insurance Service Ilsan Hospital, Goyang, ${ }^{2}$ Yonsei University College of Medicine, Seoul, Korea
Background: Patients with hemifacial microsomia may have a difficult airway due to the accompanying mandibular deformity. Fiberoptic bronchoscope-guided intubation is commonly used method for difficult airway management. However, awake fiberoptic nasotracheal intubation has several disadvantages, including difficulty in advancement of the endotracheal tube (ETT) due to the resistance between the ETT and bronchoscope. Wire-guided fiberoptic nasotracheal intubation may help to overcome the drawbacks of the conventional method.

Case: An 18-year-old man with hemifacial microsomia was scheduled for double-jaw surgery. In preoperative evaluation, he had severe retrognathia and expected difficult airway. We successfully performed wire-guided fiberoptic nasotracheal intubation combined with high-flow nasal cannula and deep sedation without any complications.

Conclusions: A guidewire may be useful, in case of difficultly in advancing the ETT or if a smaller tube is required, while performing intubation with FOB in patients with difficult airways.

Keywords: Bronchoscopes; Goldenhar syndrome; Intubation.
Hemifacial microsomia is a congenital craniofacial deformity, caused by hypoplasia of the first and second branchial arches. It affects the lower half of the face, most commonly the ear, mouth, and mandible; although a unilateral manifestation is more common, it can manifest on both sides of the face [1]. Difficulty in airway management can be expected, depending on the degree of mandibular hypoplasia, and intubation can be attempted using a fiberoptic bronchoscope (FOB), lightwand, or video laryngoscope [2]. FOB-guided intubation is the most versatile and commonly used method for difficult airway management. Moreover, successful FOB intubation, using a commercially available guide wire (Arndt ${ }^{\circledR}$ Airway Exchange catheter with J-tip guide wire, Cook Critical
Care, USA) has been reported earlier [3-8]. However, most of these cases were intubated through the laryngeal mask airway (LMA) or face mask, while wire-guided FOB nasotracheal intubation has been rarely reported. Furthermore, this kit is unavailable due to the lack of permission for its import. Herein, we report a successful wire-guided fiberoptic nasotracheal intubation in an 18-year-old patient with hemifacial microsomia who underwent double-jaw surgery.

\section{CASE REPORT}

An 18-year-old man (175 cm, $81 \mathrm{~kg})$ with right hemifacial microsomia was scheduled for double-jaw surgery, correc-

This is an Open Access article distributed under the terms of the Creative Commons Attribution Non-Commercial License (http://creativecommons.org/licenses/by-nc/4.0) which permits unrestricted non-commercial use, distribution, and reproduction in any medium, provided the original work is properly cited. 
tion with distraction osteogenesis, and mandibular anglectomy. Although he had no underlying medical history, he had undergone correction with distraction osteogenesis in 2006. At that time, the patient was classified as Cormack-Lehane grade 4, and oral intubation was performed with FOB. The patient reported a history of sleep apnea and heavy snoring, during the preoperative interview. His mouth opening was about 2.5-finger-breadth and neck extension was normal, during the preoperative physical examination. However, he had severe retrognathia and a class III Mallampati score. The thyromental distance was less than $6 \mathrm{~cm}$. Moreover, an oral panoramic view X-ray showed narrowing of the nasopharyngeal airway, due to severe retrognathia (Fig. 1). According to El-Anwar et al. [9], the mean depth (anteroposterior diameter) of the nasopharynx in normal adults is $21.8 \pm 4.6 \mathrm{~mm}$.

However, the depth of nasopharynx in our patient was 15.5 $\mathrm{mm}$, which was narrower than usual. We explained the possibility of a difficult airway and informed the patient about the special risks and procedures pertaining to performing an awake intubation procedure. Other preoperative laboratory examinations, chest X-ray, and electrocardiography revealed no abnormalities.

On the day of surgery, we prepared a Glidescope ${ }^{\oplus}$; LMA, fiberoptic bronchoscope (Olympus Optical, Japan); a 0.035-inch-thick, 145-cm-long straight-tipped Angio Guidewire (Lunderquist-ring, Cook Critical Care, Fig. 2); and a high-flow nasal cannula (Optiflow ${ }^{\mathrm{TM}}$ ). Nasotracheal intuba-

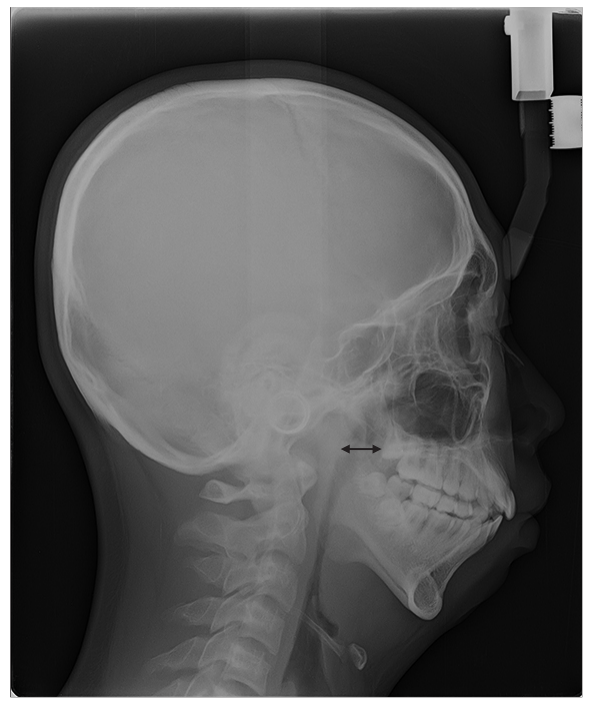

Fig. 1. Narrowing of the nasopharyngeal airway seen on the oral panoramic view X-ray (double-headed arrow). tion was required to secure space in the oral cavity for surgery; thus, we prepared nasotracheal tubes of various sizes. Non-invasive blood pressure monitoring, electrocardiogram, pulse oximetry, and bispectral index (BIS) measurement were performed in the operating room. The monitor showed an initial blood pressure of 140/84 $\mathrm{mmHg}$, a heart rate of 58 beats/min and room air saturation of $98 \%$. Lidocaine $4 \%$ was sprayed onto the tongue and oropharynx for topical anesthesia. Sufficient preoxygenation was provided with Optiflow ${ }^{\mathrm{TM}}$, at a flow rate of $20 \mathrm{~L} / \mathrm{min}$, to achieve an $\mathrm{SpO}_{2}$ of $100 \%$. We started total intravenous anesthesia (TIVA) with $2 \%$ propofol and remifentanil administered at effect-site concentrations of $2.5 \mu \mathrm{g} / \mathrm{ml}$ and $1.5 \mathrm{ng} / \mathrm{ml}$, respectively, with a targetcontrolled infusion pump. The $\mathrm{SpO}_{2}$ decreased to $96 \%$, soon after the BIS was 42 and the patient fell asleep, but there was no further desaturation, after we raised the flow rate of Optiflow $^{\mathrm{TM}}$ to $40 \mathrm{~L} / \mathrm{min}$. Since the left nostril was larger than the right nostril, owing to right-sided hemifacial microsomia, we decided to insert the FOB into the left nostril. After guiding the FOB into the left nostril of the patient, it passed into the trachea through the glottis, without any issue. We inserted the 145-cm-long, straight-tipped Angio Guidewire (Lunderquistring, Cook Critical Care) into the working channel of the FOB, till we could visualize the entrance of the guidewire into the carina (Fig. 3). We carefully removed the FOB and the guidewire remained in position. We also used the Glidescope ${ }^{\circledR}$ to confirm that the guidewire was still within the oral cavity

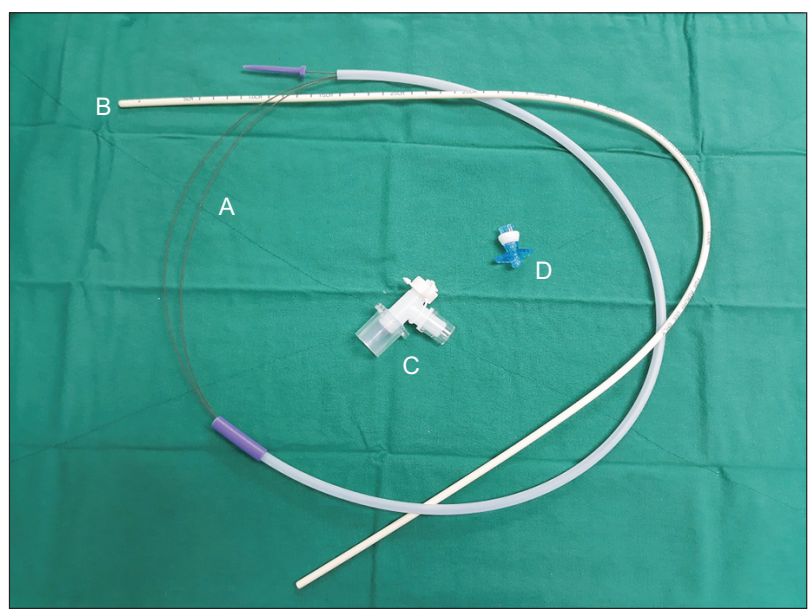

Fig. 2. Angio Guidewire and airway exchange catheter set A: straighttipped Angio Guidewire (Lunderquist-ring, Cook Critical Care), B: exchange catheter (CAEC, Cook Critical Care), C: bronchoscope airway adapter, D: Rapi-Fit ${ }^{\circledR}$ adapter. 


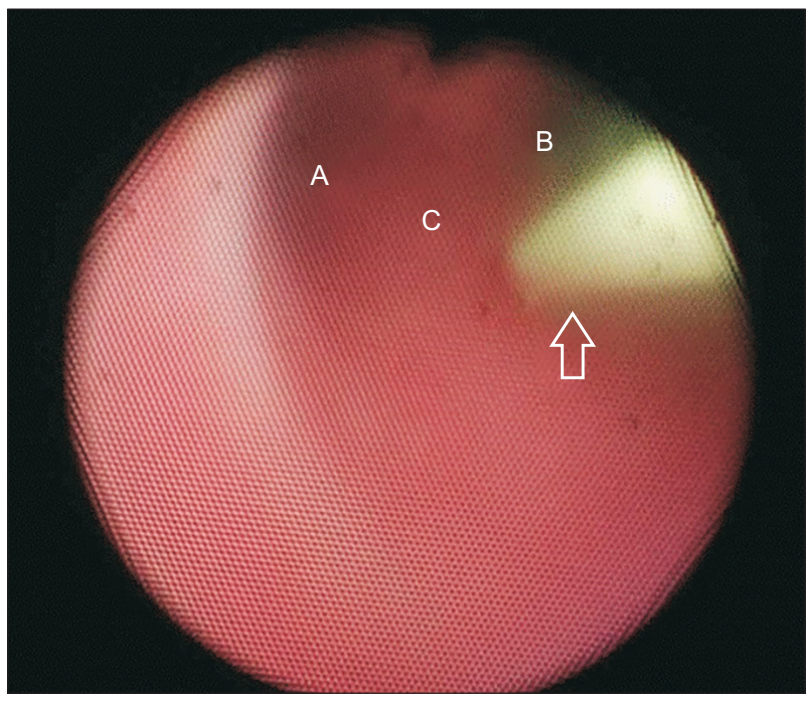

Fig. 3. The fiberoptic bronchoscope showing that the guidewire is positioned in the trachea A: left main bronchus, B: right main bronchus, C: carina, Empty arrow: guide wire.

and had passed through the vocal cord, and into the trachea (Fig. 4). The exchange catheter (1.6-mm internal diameter [ID], 2.7-mm outer diameter, CAEC, Cook Critical Care) was inserted over the Angio Guidewire and the Angio Guidewire was pulled out. A nasotracheal tube with $6.5 \mathrm{~mm}$ ID was advanced carefully over the exchange catheter into the airway without incidence. After removing the exchange catheter, the tracheal tube cuff was inflated, and proper positioning of the ETT was confirmed by auscultating both lungs and by confirming continuous positive end-tidal $\mathrm{CO}_{2}$. The monitor showed a blood pressure of 133/86 $\mathrm{mmHg}$, heart rate of 95 beats/min, an $\mathrm{SpO}_{2}$ of $97 \%$, and a BIS of 50 after intubation. An oxygenation level of $97 \%$ or higher was maintained throughout the procedure. After confirming proper intubation, we adjusted the effect-site concentration of propofol and remifentanil to $4.0 \mu \mathrm{g} / \mathrm{ml}$ and $2.5 \mu \mathrm{g} / \mathrm{ml}$, respectively, and administered $80 \mathrm{mg}$ of rocuronium intravenously. The time required for the administration of intubation to its confirmation was $16 \mathrm{~min}$. The intra and postoperative periods were uneventful.

After the procedure, we provided a full explanation regarding this case report to the patient and obtained permission for the same.

\section{DISCUSSION}

The management of patients with difficult airways is a

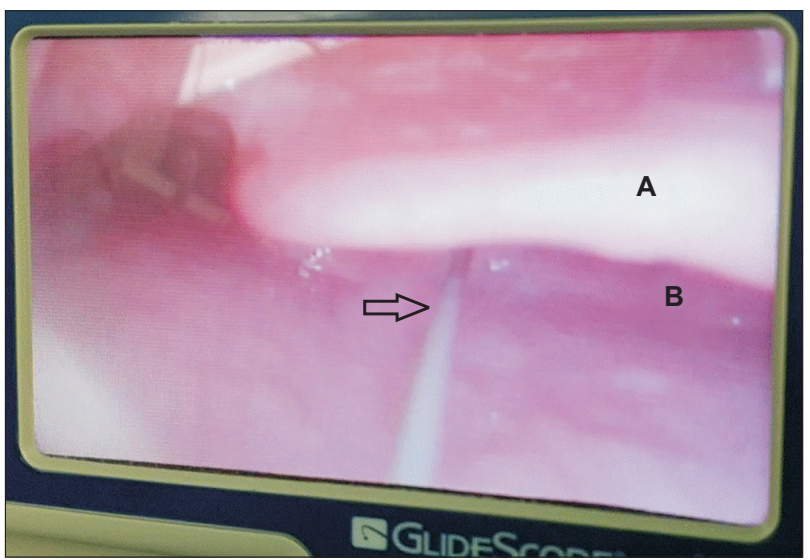

Fig. 4. The guidewire passing through the vocal cord and into the trachea as seen with the Glidescope ${ }^{\circledR}$ A: epiglottis, B: arytenoid cartilage, Empty arrow: guide wire.

constant source of dilemma for anesthesiologists and special care and attention are essential during the preparation phase of anesthesia for these patients. Patients with an anticipated difficult airway for intubation should be interviewed before surgery for any previous history of airway difficulty and a detailed physical examination should be conducted. Moreover, a variety of equipment for difficult airway management should be prepared, including a video laryngoscope, lightwand, supraglottic airway devices, and flexible FOB intubation equipment. Awake intubation using FOB should be considered, because it is a common method for difficult airway management, and was successful in $88-100 \%$ of patients with difficult airways, according to earlier studies [10].

However, the ETT must be preloaded onto the bronchoscope, while performing fiberoptic nasal intubation, which can cause several problems. There is a limit to the size of the ETT, which can be used because the ETT must be pre-loaded onto the FOB. For example, the ETT size available for FOB with a suction port should have an ID of $5.5 \mathrm{~mm}$ or more. Moreover, resistance may be felt, while advancing the ETT, despite sufficient lubrication; difficulty in deciding the appropriate size of the ETT, due to unexpected turbinate hypertrophy or deviated nasal septum; and difficulty in passing the ETT through the vocal cords over the bronchoscope, because of interference by the glottis structures $[11,12]$. The preloaded ETT and FOB should be removed in such cases and replaced with a smaller ETT and the procedure should be repeated. This can result in nasal mucosal bleeding, edema, and traumatic laryngeal injury, which can make visualization through the FOB and subsequent attempts with ETTs of other sizes 
more challenging [12]. However, wire-guided fiberoptic nasotracheal intubation may possibly overcome the above-mentioned drawbacks, by allowing more time to safely secure the difficult airway. Naithani and Jain [12] reported a case where the guidewire was used as the rescue technique, after the first attempt at bronchoscopic nasotracheal intubation failed.

Generally, a guidewire is included in a commercially available airway-exchange set or retrograde intubation set for use in difficult airway management $[4,8]$. A separate Arndt $^{\circledR}$ Airway Exchange catheter with a J-tip guidewire (Cook Critical Care) is available in the USA, which includes a $14.0 \mathrm{Fr} / 70 \mathrm{~cm}$ catheter and a 160-cm long guidewire. This set is intended for intubation with the LMA conduit using a FOB, bronchoscopic port, J-tip guidewire, and exchange catheter. According to Heier et al. [3], once the guidewire and exchange catheter are positioned in the trachea, a tracheal tube of $5.0-\mathrm{mm}$ or greater ID can be inserted. If a tracheal tube with a smaller ID is desired, intubation can be performed by threading the ETT over the guidewire alone [3]. However, because the Arndt ${ }^{\circledR}$ Airway Exchange catheter with J-tip guide wire has not yet been introduced in Korea, it may not be easy to use routinely in the same manner as previously reported cases.

In our case, narrowing of the upper airway and difficulties with tracheal intubation were expected owing to severe retrognathia in the patient with hemifacial microsomia. Preoperative evaluation should be performed with imaging tests such as computed tomography (CT), for patients who are expected to have difficult airways. It is necessary to prepare an endotracheal tube suitable for the patient's airway. However, CT was not available to confirm the airway preoperatively in this case. Our team was at fault for proceeding with the surgery without accurate airway evaluation. After the patient entered the operating room, we determined which endotracheal tube would pass through the patient's nostrils. We decided to try $6.5-\mathrm{mm}$ tube first, considering the height of the patient. If the $6.5-\mathrm{mm}$ tube could not be advanced, we attempted to replace it with a smaller tube (through the guidewire). Hence, we prepared to perform wire-guided fiberoptic nasal intubation for the patient using a $145-\mathrm{cm}$ long straight-tipped Angio Guidewire (Lunderquist-ring, Cook Critical Care). Although this guidewire is not generally used for airway exchange, it is made from polytetrafluoroethylene-coated stainless steel, the same as the Amplatz guidewire (which is a J-tip guidewire), included in Arndt ${ }^{\circledR}$ Airway Exchange catheter set (Cook Criti- cal Care). Its diameter is also similar to that of the Amplatz guidewire ( $0.038 \mathrm{inch})$. Therefore, it could easily pass through the working channel of the FOB in this case. Moreover, the length of the guidewire was adequate for nasotracheal intubation and the 6.5-mm ID nasal ETT was successfully passed along the guidewire in the first attempt. If the tip of guidewire reaches the carina via FOB, additional confirmation of position is unnecessary. Since the Glidescope was prepared in advance for difficult airway management in this case, it was used to verify the guidewire's position (Fig. 4). The patient was sufficiently sedated via TIVA and the mouth opening was 2.5 finger-breadth, and thus, there was no issue with Glidescope blade insertion.

Moreover, TIVA and Optiflow ${ }^{\mathrm{TM}}$ can simplify the awake FOB intubation procedure. We administered TIVA during the procedure, and the patient was not conscious nor anxious, as BIS was maintained within the range of $40-50$. Optiflow $^{\mathrm{TM}}$ ensured that the patient's oxygenation was maintained above $97 \%$, even though he ventilated spontaneously and was sedated deeply. Optiflow ${ }^{\mathrm{TM}}$ can supply 50-70 L of oxygen per minute and humidified warm air prevents damage to the nasal mucosa due to high flow. Moreover, the Optiflow ${ }^{\mathrm{TM}}$ nasal cannula is soft and occupies less than half of the area of the nostrils, allowing for continuous oxygenation and simultaneous passage of the fiberscope and tracheal tube. Recent studies have reported that providing sufficient oxygenation using Optiflow ${ }^{\mathrm{TM}}$, helps to prevent oxygen desaturation during awake FOB intubation [13,14].

In conclusion, a difficult airway is one of the biggest challenges faced by an anesthesiologist in the operating room. It is necessary to prepare solutions when a difficult airway is anticipated, or the conventional intubation technique fails. Moreover, knowledge of the different ways of securing a difficult airway is beneficial, as one method may not be suitable for all situations, depending on the patient's condition. A guidewire may be useful, in case of difficultly in advancing the ETT or if a smaller tube is required, while performing intubation with FOB in patients with difficult airways. Moreover, if a commercially available airway-exchange set is not available, other types of guidewire can be used as an alternative, as demonstrated by our case. 


\section{CONFLICTS OF INTEREST}

No potential conflict of interest relevant to this article was reported.

\section{ORCID}

Seon Ju Kim, https://orcid.org/0000-0002-1911-8143

Haeyeon Lee, https://orcid.org/0000-0001-8065-9590

Seung Yeon Choi, https://orcid.org/0000-0002-9620-3499

\section{REFERENCES}

1. Mielnik-Błaszczak M, Olszewska K. Hemifacial microsomiareview of the literature. Dent Med Probl 2011; 48: 80-5.

2. Nargozian C. The airway in patients with craniofacial abnormalities. Paediatr Anaesth 2004; 14: 53-9.

3. Heier JM, Schroeder KM, Galgon RE, Arndt GA. Wire-guided (Seldinger technique) intubation through a face mask in urgent, difficult and grossly distorted airways. Saudi J Anaesth 2012; 6: 292-4.

4. Arndt GA, Topp J, Hannah J, McDowell TS, Lesko A. Intubation via the LMA using a Cook retrograde intubation kit. Can J Anaesth 1998; 45: 257-60.

5. Cooper JR Jr. Use of a LMA and a sequential technique for unanticipated difficult intubations. Anesthesiology 2002; 97: 1326.

6. Rajan GR. Fiberoptic wire-guided transoral and through the LMA intubation technique using modified gum elastic bougie.
Anesth Analg 2005; 100: 599-600.

7. Warrillow S. Difficult intubation managed using standard laryngeal mask airway, flexible fibreoptic bronchoscope and wire guided enteral feeding tube. Anaesth Intensive Care 2005; 33 : 659-61.

8. Joffe AM, Arndt G, Willmann K. Wire-guided catheter exchange after failed direct laryngoscopy in critically ill adults. J Clin Anesth 2010 ; 22: 93-6.

9. El-Anwar MW, Ali AH, Elnashar I, Elfiki IM, Ahmed AF, Abdulmonaem G. Normal nasopharyngeal measurement by computed tomography in adult. J Craniofac Surg 2017; 28: e395-8.

10. Apfelbaum JL, Hagberg CA, Caplan RA, Blitt CD, Connis RT, Nickinovich DG, et al. Practice guidelines for management of the difficult airway: an updated report by the American Society of Anesthesiologists Task Force on Management of the Difficult Airway. Anesthesiology 2013; 118: 251-70.

11. Paul A, Nathroy A. Awake fiberoptic nasal intubation in a patient scheduled for commando's operation. Int J Health Allied Sci 2017; 6: 123-6.

12. Naithani M, Jain A. Failed nasal intubation after successful flexible bronchoscopy: guide wire to the rescue. J Anaesthesiol Clin Pharmacol 2011; 27: 395-7.

13. Badiger S, John M, Fearnley RA, Ahmad I. Optimizing oxygenation and intubation conditions during awake fibre-optic intubation using a high-flow nasal oxygen-delivery system. Br J Anaesth 2015; 115: 629-32.

14. Berlin D, Singh I, Barjaktarevic I, Friedman O. A technique for bronchoscopic intubation during high-flow nasal cannula oxygen therapy. J Intensive Care Med 2016; 31: 213-5. 